Analog Integrated Circuits and Signal Processing, 14, 9-28 (1997)

(c) 1997 Kluwer Academic Publishers, Boston. Manufactured in The Netherlands.

\title{
Selection of Voltage Thresholds for Delay Measurement
}

\author{
V. CHANDRAMOULI AND KAREM A. SAKALLAH
}

EECS Department, The University of Michigan, Ann Arbor, MI 48109-2122

Received June 24, 1996; Accepted January 22, 1997

\begin{abstract}
Since all physical devices have a finite non-zero response time, the notion of delay between the input and output logic signals arises naturally once digital abstraction is done. This delay should be positive and non-zero, since a physical device takes a finite amount of time to respond to the input. Defining a strictly positive delay is not a problem in the abstract domain of logic signals, since input and output "events" are precisely defined. However, when the signal non-idealities are accounted for, the notion of events is blurred and it is not obvious how to define delay such that it reflects the causal relationship between the input and the output. By necessity, we define the start and end points of these events by determining the time instants when the signals cross some appropriate voltage thresholds. The selection of these voltage thresholds for logic gates as well as simple interconnect wires, is the subject of this paper. We begin by a discussion of what we mean by signal delay and how it arises in a logic gate. With this background, starting from ideal inputs to ideal inverters and concluding with physical inputs to physical inverters, we examine the problem of threshold selection for inverters through a logical sequence of model refinement, using a combination of analytical and experimental techniques. Based on the insight gained through this analysis, we examine the problem for multi-input (both static and dynamic) gates as well as point-to-point interconnect wires. We show that thresholds derived from the gate's DC voltage transfer characteristic removes the anomalies, such as negative delay and large sensitivity to input waveshape effects, that can arise with the widely used 50\% and 10\%-90\% thresholds. Despite its fundamental nature, however, we note that the problem of threshold selection has received scant attention in the literature. To the best of our knowledge, this is the first detailed study of this problem.
\end{abstract}

Key Words: thresholds, delay, delay measurement, VTC, transition time, interconnect, causality

\section{Introduction}

Logic gates and the interconnect wires used for implementing a digital circuit are inherently analog devices that operate on continuous voltage and current waveforms (see Figure 1-1(a)). In order to specify and design digital systems, these continuous waveforms are abstracted as step (0-1 or logic) waveforms (Figure 11(b)). At the functional level, such an abstraction facilitates the use of the mathematical machinery of Boolean algebra to formally specify and design the digital system. In addition, such an abstraction establishes a temporal relationship between the inputs and output of a gate that is captured by the intuitive notion of signal delay. For example, the output of the inverter in Figure 1-1(b) changes at time $t_{1}+\Delta$ in response to an input change at $t_{1}$ and the inverter is said to have a delay of $\Delta$. It is important to note that such a delay is an expression of causality between two related events and must, thus, be strictly positive. When dealing with step inputs and outputs, there is no ambiguity in determining when the triggering event at the input and the resultant event at the output occur. Delay in such an ideal world is, accordingly, a well-defined concept.

When dealing with continuous signals it is not immediately obvious when "events" occur or how to define a meaningful delay between such signals. Appealing to the digital nature of the signals under consideration, it is reasonable to preserve the notion of events. Unlike the ideal events of step signals, however, these "real" events are non-instantaneous occurrences that span a finite time interval. This distinction between ideal and real events has several major implications:

- Whereas ideal events are unambiguously identified with the time instant corresponding to an abrupt change in signal value, identifying real events requires the selection of appropriate voltage thresholds that delimit the "significant" portion of a signal. We will refer to these thresholds as the low and high thresholds $V_{l}$ and $V_{h}$ and 


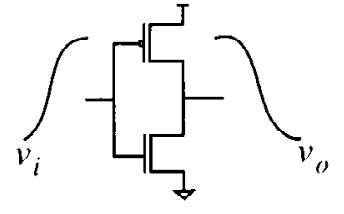

(a) Actual circuit and waveforms

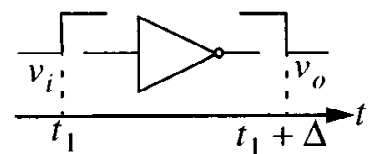

(b) Digital abstraction

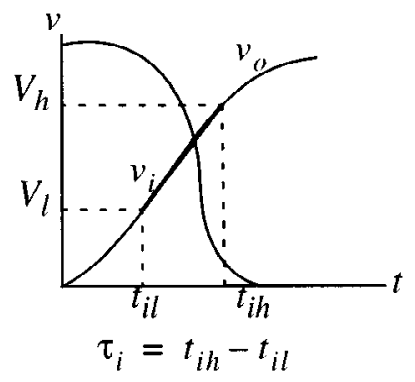

(c) The definition of signal transition time

Fig. 1-1. The notion of events and signal delay.

to the voltage interval $\left[V_{l}, V_{h}\right]$ as the transition region.

- The finite duration of an event indicates how long it takes a signal to traverse the transition region, and will be referred to as the signal transition or switching time $\tau$ (see Figure 1-1(c)). When it is necessary to distinguish the transition direction, this duration may be labeled as the rise or fall time.

- Whereas the choice of reference times for measuring delay between two related non-instantaneous events may not be obvious, any choice of reference times will yield a delay that is a function of the input transition time $\tau_{i}$. Delay functions will, thus, have the general form $\Delta=f\left(\tau_{i}\right.$, circuit and process parameters).

Our goal in this paper is to examine how signal delay is affected by the choice of thresholds used to define it. We study this problem for single- and multi-input gates as well as for simple interconnect structures. This undertaking has both theoretical and practical importance since an improper choice of thresholds can lead to anomalies such as negative delay. For instance, the popular 50\% threshold, first suggested in [7], has been shown by several researchers $[1,5,6,18,20,21]$ to result in negative delay. Other thresholds, such as the $10 \%-90 \%$, first proposed in [14], have no theoretical justification and artificially exaggerate the effect of input waveshape on delay. Anomalies related to multi- input gates result not only from poor threshold choices but also from incorrect identification of the "dominant" input; referencing delay measurement to either the first or last changing input can be shown to violate causality.

This paper is organized in five sections as follows. In Section 2, we analyze the problem of threshold selection for inverters, starting from an ideal model of the input and the gate, gradually refining it, and ending with a simulation using physical inputs and gates. Specifically, we seek to establish guidelines for selecting thresholds that preserve the notion of causality and yield strictly positive delays under all operating conditions. Further, we also address the problem of signal transition time measurement and show how the 10\%$90 \%$ threshold exaggerates the input waveshape effect on delay. In Section 3, we consider multi-input gatesboth static and dynamic. The insight gained through inverter analysis is used to address the problem of threshold selection for multi-input gates. In Section 4, we examine delay measurement for point-to-point interconnects. Finally, in Section 5, we conclude the paper with a summary of the principal results.

\section{Threshold Selection for Inverters}

Before we delve deeper into the issue of threshold selection, it is insightful to discuss the basis for digital abstraction, since delay is a natural consequence of digital abstraction. It is the existence of circuits 


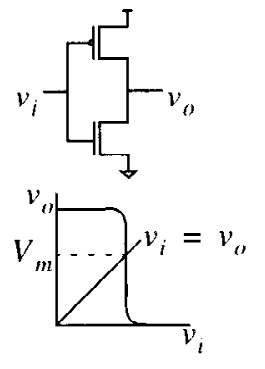

(a) Inverter and its VTC

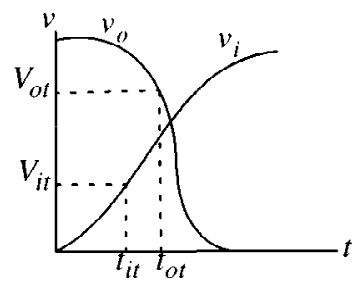

$\Delta-t_{o t}-t_{i t}$

(d) Delay measurement

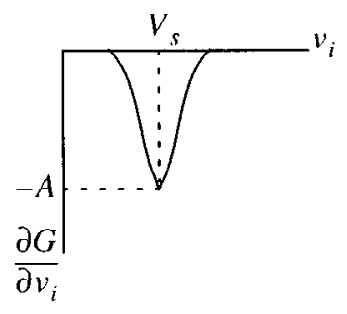

(b) Variation of gain

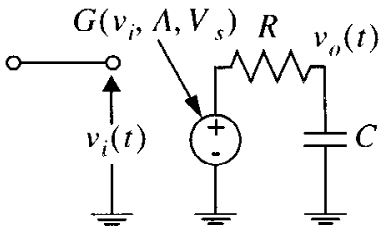

(c) Equivalent circuit of inverter

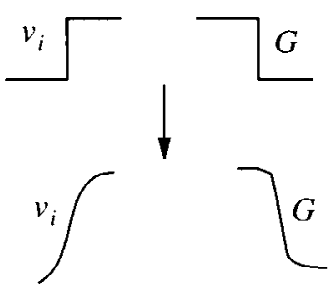

(e) Two extreme cases

Fig. 2-1. VTC and equivalent circuit of inverter.

that amplify which makes a digital abstraction possible. While some logic functions, for area/speed advantages, can be implemented using pass gates or some other "clever" circuits that do not amplify, however, levelrestoring (i.e. amplifying) circuits have to be suitably interspersed in the logic for reliable operation of the complete system. The DC input-output voltage plot, called the voltage transfer curve (or simply the VTC), of these amplifiers, such as the inverter, has the general shape of a deformed letter $\mathrm{S}$ (or its mirror image) as shown in Figure 2-1(a). The voltage gain of such circuits is a function of the input voltage and is plotted in Figure 2-1(b). The severe non-linearity exhibited by the inverter partitions the input voltage range into two wide regions where the magnitude of the gain of the amplifier is close to zero, separated by a narrow high gain region. In an ideal digital gate, the width of this narrow region collapses to a single voltage where the gain is infinite, and the gain is zero elsewhere. The existence of these two wide regions where the gain is zero enables us to assign logic levels to these regions and thus makes a digital abstraction possible. Referring to Figure 2-1(a) and (b), we denote the unity DC gain voltage, when $v_{i}=v_{o}$, by $V_{m}$, the magnitude of the maximum gain by $A$, and the voltage at which the gain is maximum by $V_{s}$. We base the selection of thresholds on the VTC of the circuit since it makes the development sufficiently general and the conclusions valid for any technology.

In order to study the dynamic behavior of the inverter, we introduce the equivalent circuit shown in Figure 2-1(c). The VTC is represented by a voltagecontrolled-voltage-source (VCVS), $G$, parametrized by $A$ and $V_{s}$; the resistance $R$ models the output impedance of the inverter and $\mathrm{C}$ models the load capacitance as well as the non-linear transistor capacitances of the inverter. The governing differential equation for the output voltage, $v_{o}(t)$, of the inverter is then:

$$
R C \frac{d v_{o}}{d t}+v_{o}=G\left(v_{i}(t), V_{s}, A\right)
$$

A typical input stimulus and the output response of the inverter is shown in Figure 2-1(d). We denote the time instant when the input crosses the input threshold, $V_{i t}$ by $t_{i t}$ and the time instant when the output crosses the output threshold, $V_{o t}$, by $t_{o t}$. We note that $V_{i t}$ and $V_{o t}$ are merely placeholders for actual voltage values which could lie anywhere in the inverter's voltage swing. Referring to the figure, the delay of the 
inverter is given by the following template:

$$
\Delta=v_{o}^{-1}\left(V_{o t}\right)-v_{i}^{-1}\left(V_{i t}\right)
$$

In the following subsections, we examine this behavior of delay for different choices of $V_{i t}$ and $V_{o t}$, by solving (2.1) for different input and VTC shapes. As shown in Figure 2-1(e), at one end of this spectrum is the ideal input interacting with an ideal gate whereas at the other end is the actual signal interacting with the actual VTC. However, as mentioned earlier, when the input is modeled as a step, there is no ambiguity about the occurrence of the input event and a positive delay can be unequivocally defined. Therefore, as a first step towards approaching realistic waveforms, we model the input by a saturated ramp. In subsection (2.1), we consider a ramp input to an ideal inverter, followed by an analysis of a ramp input to an inverter with a piecewise-linear VTC (the first step towards modeling a physical inverter) in subsection (2.2). In subsection (2.3), the piecewise-linear model of the inverter VTC is refined to a continuous non-linear VTC to mimic the physical VTC and the conclusions reached in subsection (2.2) are confirmed through numerical simulation. Finally, in subsection (2.4), we reconfirm our results using a circuit simulator, for physical signals and VTCs. In the mathematical analysis of subsections (2.1)-(2.3), we assume that all voltages are normalized to lie within $[0,1]$ and that all times are normalized with respect to the circuit time constant $R C$. Further, we consider only a rising signal at the inverter input since the reasoning for a falling input is similar.

\subsection{A Ramp Input to an Ideal Inverter}

As a first step towards modeling an actual signal, we consider a saturated ramp given by:

$$
v_{i}(t)=\left\{\begin{array}{l}
\frac{t}{\tau_{i}}, 0 \leq t \leq \tau_{i} \\
1, t>\tau_{i}
\end{array}\right.
$$

This signal ${ }^{1}$ is applied to the input of an ideal inverter (see Figure 2-2(a)) whose VTC is shown in Figure 22(b). Note that for ideal gates we have $V_{s}=V_{m}$, and the gain, when $v_{i}=V_{m}$, is infinite as shown in Figure 22(c). It is easy to see that by composing the ramp input with the ideal VTC, the VCVS in the equivalent circuit, $G\left(v_{i}(t), V_{m}\right)$, is a step as shown in Figure 2-2(d). Thus, even though the input signal is not ideal, the ideal VTC serves to transform it into an ideal signal. The output response, on solving equation (2.1) with the given input and VTC waveshapes [16], is shown in Figure 2-2(e). The equation for delay (see (2.2)) in this case is given by:

$$
\Delta=\tau_{i}\left(V_{m}-V_{i t}\right)-\ln \left(V_{o t}\right)
$$

Referring to Figure 2-2(e), intuitively, an ideal gate implies a choice of $V_{i t}=V_{m}$, since it is only when the input voltage reaches $V_{m}$ that it causes an output change to occur. With this choice of $V_{i t}$, from (2.4) it is clear that delay is positive for any choice of $V_{o t}$ such that it lies within $[0,1]$; only the magnitude of delay would vary depending on the choice of $V_{o t}$. It is also evident that the delay is independent of $\tau_{i}$. For $V_{i t}<V_{m}$, delay is still positive and monotonically increasing with $\tau_{i}$; however, we reject this choice since it overestimates delay by incorrectly accounting for causality (since there is no change in the output when $V_{i t}<V_{m}$ ) and also produces an artificial increase in delay with input slope. Similarly, we reject a choice of $V_{i t}>V_{m}$ since it underestimates delay and could result in negative delay for slow inputs. The behavior of delay for these different choices of thresholds is shown in Figure 2-2(f).

Thus, in summary, the choice of delay thresholds for ideal gates, even when excited by non-ideal inputs, is unambiguous with $V_{i t}=V_{m}$ and $V_{o t} \in[0,1]$. With this choice of delay thresholds, delay is also independent of the input transition time. However, this does not lead to a consistent choice of delay thresholds. To see why, consider another ideal inverter connected to the output of the inverter under consideration, as shown in Figure 2-2(g). This inverter has a falling transition at its input and going through a similar analysis, we find that we need $V_{i t}=V_{m}$. Therefore, in order to define delay meaningfully for the combination, we need to choose $V_{o t}=V_{m}$. Thus, for ideal gates we are left with only one reasonable choice of thresholds: $V_{i t}=V_{m}$ and $V_{o t}=V_{m}$.

\subsection{A Ramp Input to an Inverter with Piecewise- Linear VTC}

Next we consider a ramp input to an inverter with a piecewise-linear VTC as shown in Figure 2-3(a). The VTC is given by the following set of equations and is 


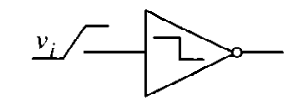

(a) Ramp input to an ideal inverter

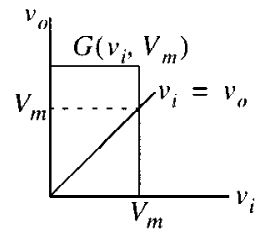

(b) An ideal VTC

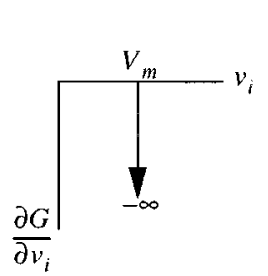

(c) Variation of gain

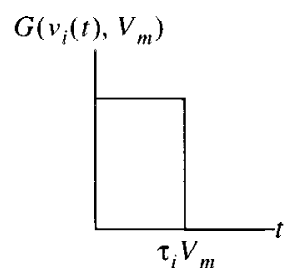

(d) Conversion of ramp to step through ideal VTC

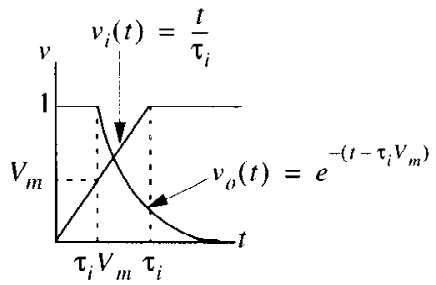

(c) The output of the ideal inverter

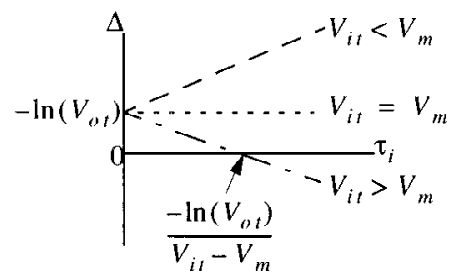

(t) Different choices of thresholds

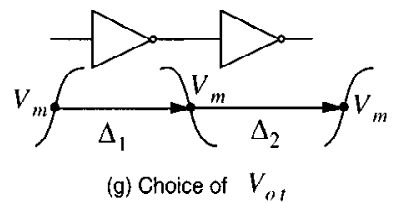

Fig. 2-2. A ramp input to an ideal inverter.

shown in Figure 2-3(b):

$$
\begin{aligned}
& G\left(v_{i}, A, V_{s}\right)= \\
& \left\{\begin{array}{l}
1, v_{i}<V_{i l} \\
\left(\frac{\left(V_{i h}-v_{i}\right)}{V_{i h}-V_{i l}}=A\left(V_{i h}-v_{i}\right)\right), V_{i l} \leq v_{i} \leq V_{i h} \\
0, v_{i}>V_{i h}
\end{array}\right.
\end{aligned}
$$

Thus, the VTC has a finite but constant gain $A>1$ between $V_{i l}$ and $V_{i h}$ and 0 gain elsewhere, as shown in Figure 2-3(c). It is interesting to note that $V_{s}$ is not uniquely defined and can be any value between $V_{i l}$ and $V_{i h}$. Unlike the ideal VTC, on composing the ramp input with the piecewise-linear VTC, we are still left with a ramp signal for the VCVS (see Figure 2-3(d)) in the equivalent circuit. Therefore, it is not immediately obvious what choice of delay thresholds would result in a causal definition of delay. The resulting output waveform obtained by solving (2.1) with the given input and VTC parameters is shown in Figure 2-3(e) and consists of two distinct parts.

As a first step towards the selection of thresholds for non-ideal gates, we continue with choices made for ideal gates $\left(V_{i t}=V_{m}\right.$ and $\left.V_{o t}=V_{m}\right)$ and investigate the behavior of delay. However, since the output response is composed of two different waveshapes, it is not possible to write an explicit equation for delay. Instead, we break the analysis into two parts: the behavior of delay as $\tau_{i} \rightarrow 0$ (fast inputs) and as $\tau_{i} \rightarrow \infty$ (slow inputs).

Fast inputs: For small values of $\tau_{i}, V_{B}$ is close to 1 and can be approximated as:

$$
V_{B}=1-\frac{\tau_{i}}{2 A}
$$




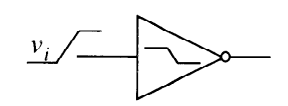

(a) Ramp input to inverter with linear VTC
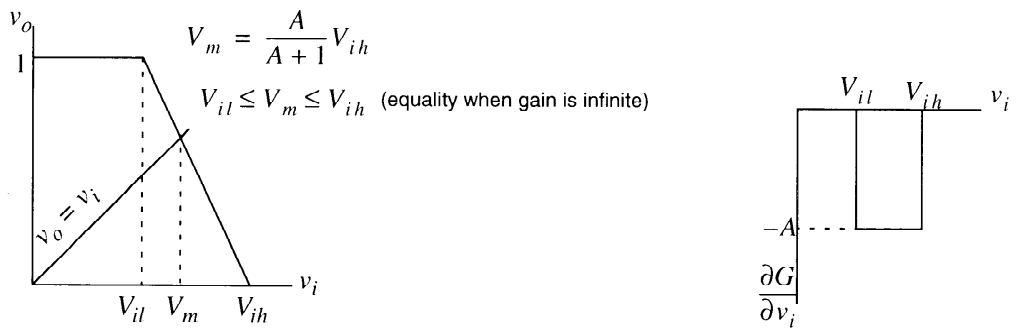

(b) The piecewise-linear VTC

(c) Variation of gain

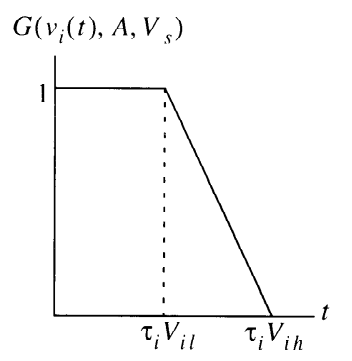

(d) Composition of ramp input with VTC

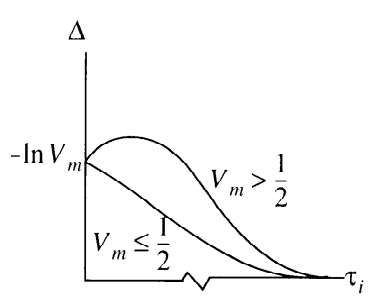

(f) Delay with $V_{i t}=V_{o t}=V_{m}$

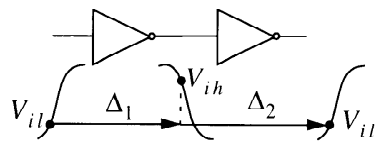

(g) Choice of $V_{o t}$

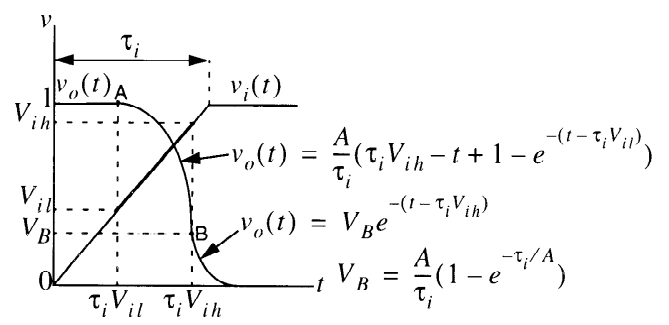

(e) The output waveform

Fig. 2-3. Ramp input to an inverter with piecewise-linear VTC.

and assuming $V_{B} \geq V_{m}$, the output voltage is given by:

$$
v_{o}(t)=\left(1-\frac{\tau_{i}}{2 A}\right) e^{-\left(t-\tau_{i} V_{i h}\right)}
$$

and the delay is given by:

$$
\Delta=\tau_{i} V_{i h}-\ln \left(\frac{V_{o t}}{1-\frac{\tau_{i}}{2 A}}\right)-\tau_{i} V_{i t}
$$

It is obvious from this equation that the delay is positive for a step input and is given by $-\ln V_{o t}$ (which confirms our observation with ideal gates and ideal inputs; see Figure 2-2(f)). In order to see its behavior with increasing $\tau_{i}$, we differentiate (2.8) to get:

$$
\frac{d \Delta}{d \tau_{i}}=V_{i h}-V_{i t}-\frac{1 /(2 A)}{1-\tau_{i} /(2 A)}
$$

It follows therefore for $\left.\frac{d \Delta}{d \tau_{i}}\right|_{\tau_{i}=0}>0$ we must satisfy:

$$
V_{i t}<V_{m}+\frac{\left(2 V_{m}-1\right)}{2 A}
$$

where $V_{i h}$ has been expressed in terms of $V_{m}$ (see the relation in Figure 2-3(b)). Substituting $V_{i t}=V_{m}$ in the above inequality we find that delay increases with increasing input transition time if $V_{m}>0.5$ and decreases otherwise. It is also clear that the choice of 
output threshold does not affect the monotonicity of delay; it only affects the value of delay for step inputs.

Slow inputs: For large values of $\tau_{i}, V_{B}$ is close to 0 and assuming $V_{B} \leq V_{m}$, the output voltage is given by:

$$
v_{o}(t)=\frac{A}{\tau_{i}}\left(\tau_{i} V_{i h}-t+1-e^{-\left(t-\tau_{i} V_{i l}\right)}\right)
$$

At the instant $t_{o t}$, the output voltage $V_{o t}$ is given by:

$$
\begin{aligned}
V_{o t} & =A V_{i h}-A \frac{t_{o t}}{\tau_{i}}+\frac{A}{\tau_{i}}-\frac{A}{\tau_{i} e^{\left(t_{o t}-\tau_{i} V_{i l}\right)}} \\
& \cong A\left(V_{i h}-\frac{t_{o t}}{\tau_{i}}\right)
\end{aligned}
$$

Solving for delay, we have:

$$
\Delta=\tau_{i}\left[\left(V_{m}-V_{i t}\right)+\frac{1}{A}\left(V_{m}-V_{o t}\right)\right]
$$

where again $V_{i h}$ has been expressed in terms of $V_{m}$. For a choice of $V_{i t}=V_{o t}=V_{m}$, we find that the delay asymptotes to zero as $\tau_{i}$ tends to infinity.

We can summarize the discussion for this choice of thresholds in Figure 2-3(f), where the behavior for both fast and slow inputs is shown. It is not surprising that this choice of delay thresholds leads to an anomalous behavior of delay. Referring to Figure 2-3(e), observe that the output has already started to change by the time the input reaches $V_{m}$. Relating this to our discussion in the Introduction, we are incorrectly assuming the start of input event at the instant the input crosses $V_{m}$ and thus underestimate delay which results in a zero value of delay for slow inputs.

From (2.13), it is obvious that delay will be positive and increasing with $\tau_{i}$ for slow inputs when $V_{i t}<V_{m}$ and $V_{o t}=V_{m}$. While we could choose any $V_{i t}$ such that it is less than $V_{m}$, we find that a choice of $V_{i t}=V_{i l}$ supports the notion of causality. Again referring to Figure 2-3(e), we find that it is only when the input crosses $V_{i l}$ that the output starts to change. By substituting this choice in (2.10), we find that the delay is indeed positive and an increasing function of $\tau_{i}$ for fast inputs as well. Thus, a choice of $V_{i t}=V_{i l}$ and $V_{o t}=V_{m}$ appears to be satisfactory, as long as we are considering a single inverter. However, for a combination of two inverters, as shown in Figure 2-3(g), for a choice of $V_{i t}=V_{i l}$ the only consistent way to define delay is to choose $V_{o t}=V_{i h}$. A quick glance at (2.10) and (2.13), shows that delay is always positive and a monotonically increasing function of $\tau_{i}$ as shown in Figure 2-3(h).

Thus, while a single threshold such that $V_{i t}=V_{o t}=$ $V_{m}$ suffices for ideal gates, we need two thresholds, $V_{i t}=V_{i l}$ and $V_{o t}=V_{i h}$ when we consider non-ideal gates. However, a single threshold choice of $V_{i t}=$ $V_{o t}=V_{m}$ has been made by some authors ([5, 13, 18, 20]), even for non-ideal gates, which as we saw leads to a non-monotonic behavior of delay with respect to input transition time. This analysis also implies that the temporally relevant part of the input waveform is when $V_{i l} \leq v_{i} \leq V_{i h}$, which is shown in bold in Figure 23(e). It is therefore reasonable to use $V_{l}=V_{i l}$ and $V_{h}=V_{i h}$ for measuring the signal transition time.

\subsection{A Ramp Input to an Inverter with a Continuous Non-linear VTC}

We next consider the ramp input applied to an inverter with a non-linear continuous VTC as shown in Figure 2-4(a). While the preceding analysis with a piecewise-linear VTC provided valuable insight into the choice of delay thresholds, the VTC of a real gate has a smooth shape. In order to mimic the wide variety of VTC shapes in real circuits, we find it convenient to modify equation (2.5) as follows:

$G\left(v_{i}, V_{s}, A\right)=\frac{1}{2}\left\{1+\tanh \left[2 A\left(V_{s}-v_{i}\right)\right]\right\}$

This function is graphed for different values of $A$ in Figure 2-4(b). The variation of gain with $v_{i}$ is plotted in Figure 2-4(c). The gain is no longer constant and varies with the input voltage. The magnitude of gain is equal to 1 when $v_{i}=V_{i l}$, reaches a maximum of $A$ when $v_{i}=V_{s}$, and decreases again reaching a value of 1 when $v_{i}=V_{i h}$. Therefore, $V_{i l}$ and $V_{i h}$ are referred to as the unity differential gain voltages [9]. It is interesting to note the behavior of $V_{m}$ with $V_{s}$ in Figure 2-4(d). We note that $V_{m}=V_{s}$ only for symmetric VTCs when $V_{s}=0.5$; for others $V_{m}$ is slightly less or greater than $V_{s}$. We also observe that though the discontinuities in the curves have disappeared, the essential features of the continuous VTC are the same as the piecewise-linear one. Therefore, we expect the conclusions reached in the preceding subsection to remain valid. In the remainder of this subsection, we restrict ourselves to symmetric VTCs since it avoids the use of $V_{s}$ in the discussion, keeping it along the same lines 


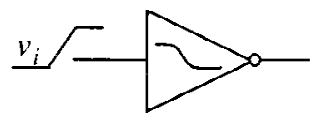

(a) Ramp input to inverter with non-linear continuous VTC

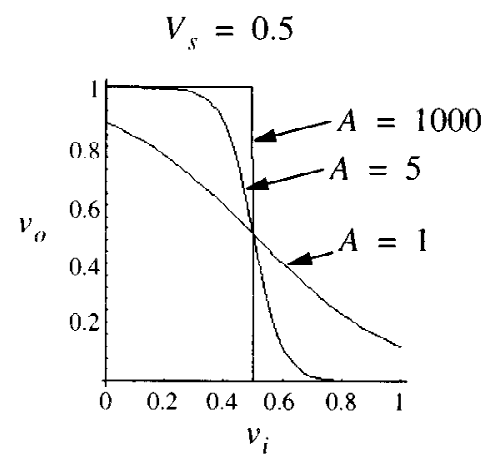

(b) VTC of an inverter for different $A$

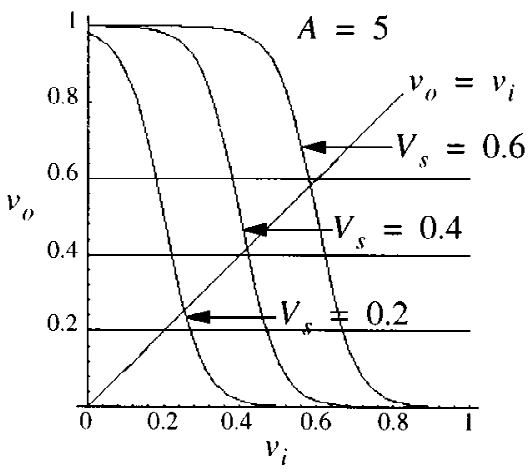

(d) VTC of inverter for different $V_{s}$

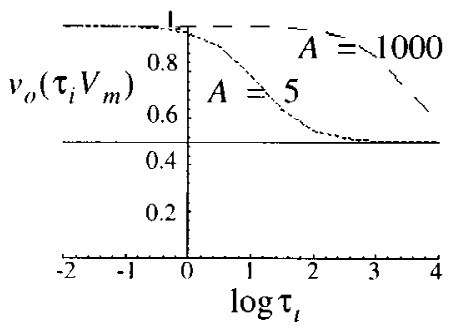

(f) The output voltage at $\tau_{i} V_{m}$

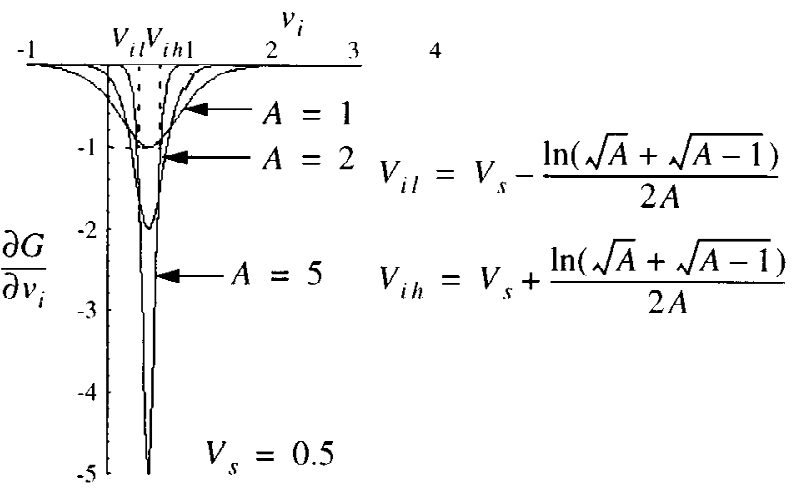

(c) Variation of gain

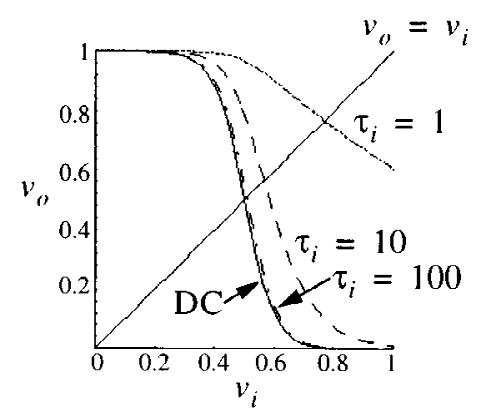

(e) The transient VTC

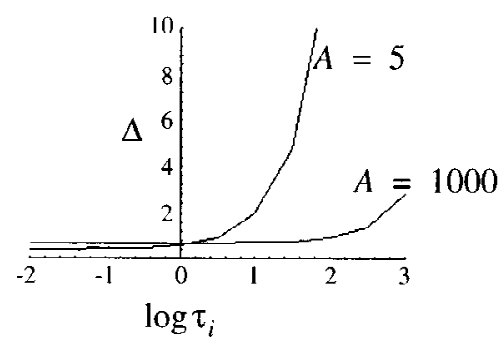

(g) Delay measured with $V_{i t}=V_{i l}$ and $V_{o l}=V_{i h}$

Fig. 2-4. Ramp input to an inverter with continuous non-linear VTC.

as before. Note that all the conclusions reached with this assumption remain valid for asymmetric VTCs as well.
Since, we cannot solve (2.1) analytically for the output voltage, we use numerical simulation using Mathematica [22] to confirm the results of the previous sub- 
section. We first show that a choice of $V_{i t}=V_{o t}=V_{m}$ leads to zero delay as $\tau_{i} \rightarrow \infty$. Since numerical methods have difficulties in determining the asymptotic behavior, we resort to an indirect technique by introducing the notion of a transient $\mathrm{VTC}^{2}$ in Figure 2-4(e). A transient VTC is obtained by numerically solving for $v_{o}(t)$ using (2.1) for a given $\tau_{i}$ and plotting it versus $v_{i}(t)$ for different instants of time, $t$. It is clear from the figure that a transient VTC approaches the DC VTC as $\tau_{i} \rightarrow \infty$. Therefore, this implies that the output voltage crosses $V_{m}$ at the same instant as the input does, resulting in zero delay. This is confirmed again in Figure 2-4(f) where we plot the output voltage at the instant the input crosses $V_{m}$, versus $\tau_{i}$, for two different gains. Note that the output voltage at this instant approaches $V_{m}$ for large $\tau_{i}$. However, also note that when the gain of the inverter is large, the delay approaches zero slower and in the limit $A \rightarrow \infty$, the delay would be a constant positive value, independent of $\tau_{i}$, confirming our analysis in subsection 2.1 for ideal gates.

However, when we choose $V_{i t}=V_{i l}$ and $V_{o t}=$ $V_{i h}$, we expect the delay to remain positive. This is because as the transient VTC approaches the DC VTC for slow inputs, the output voltage at the instant the input crosses $V_{i l}$ will approach $G\left(V_{i l}\right)$. It is easy to see from the equations for $V_{i l}$ and $V_{i h}$ in Figure 24(c) that $V_{i h}<G\left(V_{i l}\right)$. In Figure 2-4(g), we show that this choice of delay thresholds never results in negative delay and that the delay is a monotonically increasing function of $\tau_{i}$. Again, note the relative insensitivity of delay to $\tau_{i}$ when the gain of the inverter is large.

Thus, by a series of steps, starting from an ideal input to an ideal gate and concluding with a ramp input to an inverter whose VTC resembles the actual VTCs of real circuits, we have rigorously analyzed the problem of a proper choice of delay thresholds. From this analysis, it is clear that a choice of $V_{i t}=V_{i l}$ and $V_{o t}=V_{i h}$ ensures that the delay is always defined such that causality is maintained. This analysis corroborates the same choices made by other researchers [15] without a formal justification. We now confirm the results of this analysis with a physical waveform applied to a physical inverter, in the next subsection.

\subsection{Physical Input to a Physical Inverter}

While the analysis so far was based on abstract mathematical models for the inverter and the input, we need to perform experiments using physical inverters and realistic waveforms (see Figure 2-5(a)) in order to conclusively establish the results of the preceding subsections. Consider the experimental setup shown in Figure 2-5(b). In order to excite the circuit-under-test (CUT) by the characteristic waveform [3], we drive the CUT by two inverter stages, input to which is a rising ramp. The important thresholds of the CUT in Figure 25(b) are shown in the table in Figure 2-5(c), obtained through a DC simulation of the inverter. All simulations described in this subsection (and elsewhere in the paper) were performed using HSPICE [17] with the HP 0.6 $\mu \mathrm{m}$ CMOS technology [12] available through MOSIS. Unless stated otherwise, $\tau_{i}$ of the input to the CUT is measured using $V_{l}=V_{i l}$ and $V_{h}=V_{i h}$ and is changed by varying the capacitance at the input to the CUT.

We begin by showing the inappropriateness of using $V_{i t}=V_{o t}=V_{m}$ as the delay threshold for nonideal gates and compare it with a choice of $V_{i t}=V_{i l}$ and $V_{o t}=V_{i h}$. Next, we compare the transition time measurement using the $10 \%-90 \%$ thresholds versus the $V_{i l}-V_{i h}$ thresholds.

2.4.1. Delaymeasurement with $V_{i t}=V_{o t}=V_{m}$ versus $V_{i t}=V_{i l}$ and $V_{o t}=V_{i h}$. First consider the behavior of delay with $V_{i t}=V_{o t}=V_{m}$. We show the inappropriateness of this choice on two counts: 1) delay going to zero as the input becomes slow, and 2) extreme sensitivity of delay to small variations in the value of $V_{m}$. However, since we cannot show the asymptotic behavior of delay as $\tau_{i} \rightarrow \infty$ using circuit simulation, as before we resort to an indirect technique using the transient VTC. The transient VTC for the inverter is shown in Figure 2-5(d). It is obtained by plotting the output voltage versus the input voltage, at various instants of the simulation time. Since the transient VTC asymptotes to the DC VTC for slow inputs, we expect the output to cross $V_{m}$ at the same instant as the input, resulting in zero delay. This is corroborated further in Figure 2-5(e) where we show the output voltage at the instant the input crosses $V_{m}$. As shown in the figure, the output voltage at this instant asymptotes to $V_{m}$ as the input becomes slower.

Next we show the sensitivity of delay to variations in the value of $V_{m}$ in Figure 2-5(f). If the value of $V_{m}$ is incorrectly determined as $1.55 \mathrm{~V}$, delay is positive and increasing with $\tau_{i}$; however, if $V_{m}$ is incorrectly determined as $1.57 \mathrm{~V}$, the delay starts to decrease after a certain point and will eventually become negative. This 


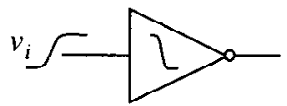

(a) Actual input to a physical inverter

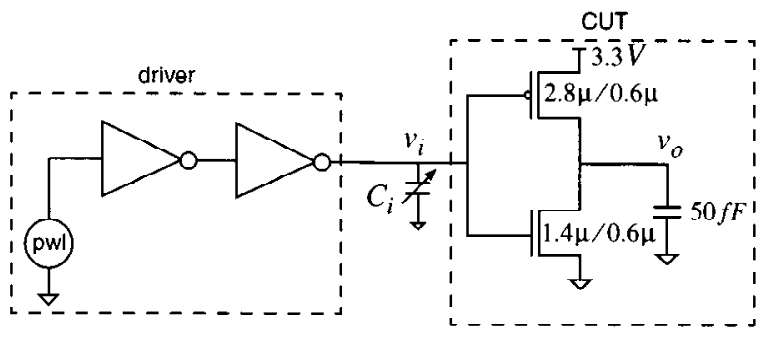

(b) Experimental setup

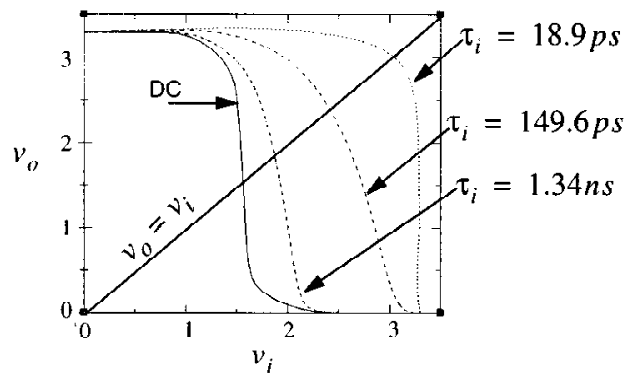

(d) Transient VTC

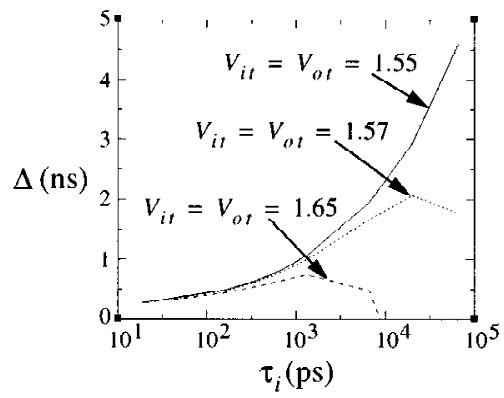

(f) Anomalous behavior of delay

\begin{tabular}{|c|c|c|}
\hline$V_{i l}$ & $V_{m}$ & $V_{i h}$ \\
\hline $1.250 \mathrm{~V}$ & $1.561 \mathrm{~V}$ & $1.746 \mathrm{~V}$ \\
\hline
\end{tabular}

(c) Thresholds of the inverter

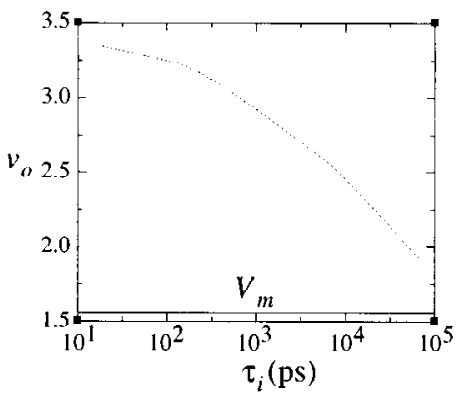

(e) $v_{0}$ at instant when $v_{i}=V_{m}$

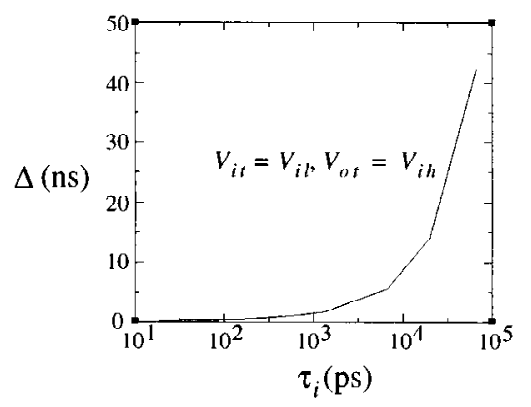

(g) Correct benavior of delay

Fig. 2-5. A physical input to a physical inverter.

result is not surprising if we refer to equation (2.13). It is clear that for $V_{i t}>V_{m}, V_{o t}>V_{m}$, the delay becomes negative and for $V_{i t}<V_{m}, V_{o t}<V_{m}$ the delay remains positive.

We also show the behavior of delay when measured using the popular 50\% threshold (which in this case is $1.65 \mathrm{~V}$ ) in Figure 2-5(f). Since for this inverter, the 50\% threshold is greater than $V_{m}$, this is indeed expected (see (2.13)). In a symmetric inverter, the 50\% threshold would equal $V_{m}$ and its behavior would be similar to the one shown in Figure 2-5(d) and (e). However, when the inverter is designed such that $V_{m}$ is greater than the $50 \%$ threshold, this choice works fine since as predicted by (2.13), the delay remains positive. 
The behavior of delay with $V_{i t}=V_{i l}$ and $V_{o t}=V_{i h}$ is shown in Figure 2-5(g). As predicted by the analysis in the preceding subsections, the delay is always positive and is a monotonically increasing function of $\tau_{i}$. Unlike the case of $V_{m}$, small variations in measuring the value of $V_{i l}$ and $V_{i h}$ will not alter the behavior of delay. Even if the $V_{i l}$ and $V_{i h}$ values are off a little bit, $V_{i t}$ will still be sufficiently smaller than $V_{m}$, and since the gain in any practical inverter is large, from equation (2.13) we can see that the delay will still be positive and increasing. Thus, the experimental results confirm our conclusion that $V_{i t}=V_{i l}$ and $V_{o t}=V_{i h}$ are the appropriate choices for measuring delay. Next we examine the behavior of signal transition time for two different choices of thresholds for measuring it.

2.4.2. Transition time measurement using the 10\%90\% thresholds versus the $V_{i l}-V_{i h}$ thresholds. If the thresholds for measuring transition time capture the temporally relevant portion of the input, then for a given input transition time, delay of the gate should depend only minimally on the specific input waveshape. We now describe an experiment we performed which shows that this is indeed the case if transition time measurement is done using $V_{l}=V_{i l}$ and $V_{h}=V_{i h}$ rather than the usual $10 \%-90 \%$ thresholds. The experimental setup used in our experiment is shown in Figure 2-6(a). We first measured the delay through the CUT with the characteristic waveform as the input. We then approximated the characteristic waveform at the input to the CUT by two different waveshapes in turn: the ramp and the exponential, and compared the new delay to the delay due to the characteristic waveform that was obtained earlier (see Figure 2-6(b)). The delay in each case was measured using the $V_{i l}-V_{i h}$ thresholds of the inverter. The approximation was done such that the ramp and the exponential had the same transition times as the characteristic waveform they replaced, for two different choices of transition time thresholds: $V_{i l}-V_{i h}$, and $10 \%-90 \%$. This experiment was then repeated a large number of times by randomly varying the parameters of the experimental setup (the range of parameters is shown in Figure 2-6(a)). The percentage error in the delay due to the waveform approximation relative to the delay due to the characteristic waveform is shown in Figure 2-6(c) for the exponential and in Figure 2-6(d) for the ramp waveshapes. The relative errors are plotted as a function of the input transition time normalized to the characteristic waveform delay.
It is clear from the scatter plots that delay is less sensitive to the input waveshape when the input transition time is measured using the $V_{i l}-V_{i h}$ thresholds rather than the $10 \%-90 \%$ threshold. It is also interesting to note that in most cases, when the input transition time is measured using the $V_{i l}-V_{i h}$ thresholds, the exponential waveshape over estimates the true delay whereas a ramp input underestimates it.

This completes our study of the problem of selecting voltage thresholds for measuring delay and transition time for a single input gate. Summarizing, we find that delay measured using the unity differential gain voltages of the gate is always positive and is a monotonically increasing function of the input transition time. Further, input transition time measured using the same thresholds results in delay that is less sensitive to the input waveshape than the conventional $10 \%-90 \%$ thresholds. We now use the insights gained from this analysis to examine the problem of threshold selection for multi-input gates in the next section.

\section{Threshold Selection for Multi-Input Gates}

\subsection{Static Gates}

Simple Gates: The situation is more complicated in a multi-input gate when many inputs switch in close temporal proximity with different transition times (see Figure 3-1(a)). Here the problem is two-fold: not only do we have to identify the voltage thresholds for measuring delay but we also need to identify the correct reference input for delay measurement. In this paper we address only the problem of choosing the correct voltage thresholds; the latter problem is tackled in [4]. As with inverters, delay measured using these thresholds must satisfy causality and must therefore yield a positive value of delay for all possible combinations of input transition times and the temporal separations between the inputs. Since the VTC played a critical part in the analysis of the inverter, we once again start with the VTC of the multi-input gate. We illustrate our approach with the three-input NAND gate shown in Figure 3-1(b).

Rather than a single VTC as in the case of an inverter, an $n$-input gate can have $2^{n}-1$ VTCs corresponding to all possible combinations of stable and switching inputs. Figure 3-1(c) shows the VTCs of the NAND gate, obtained by circuit simulation. The $V_{i l}, V_{i h}$ and $V_{m}$ of each VTC are listed in Figure 3-1(d). The VTC for the case when a is switched alone and the VTC 


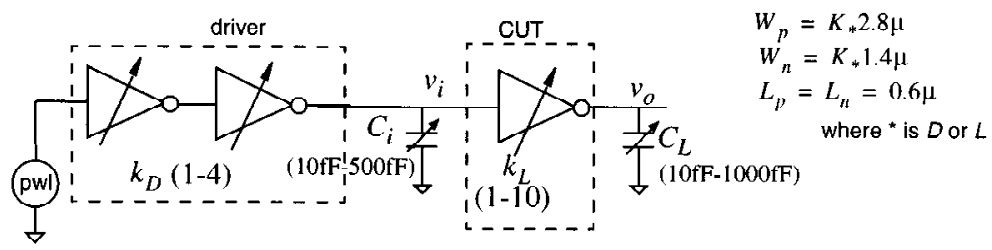

(a) Experimental setup for waveshape effects
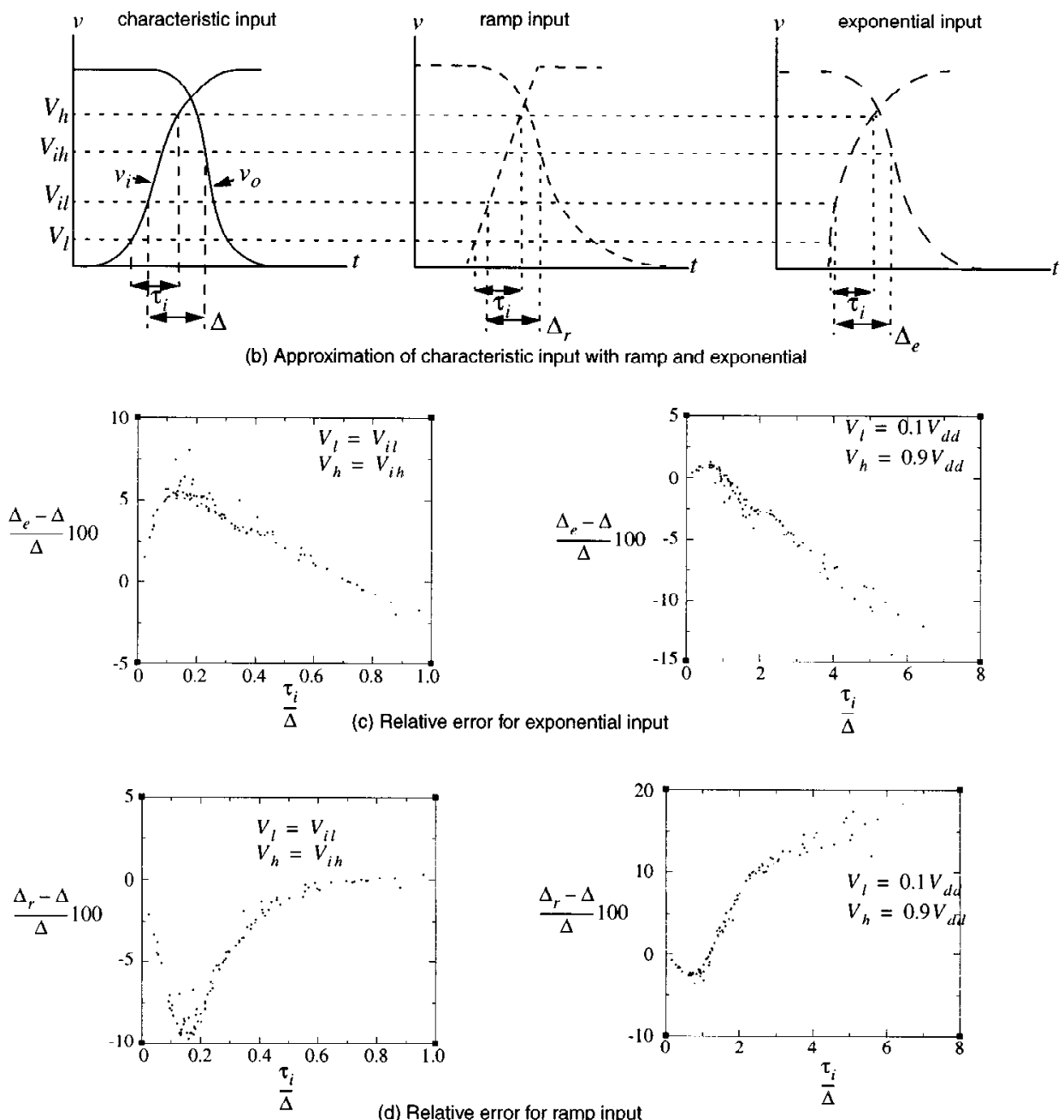

(d) Relative error for ramp input

Fig. 2-6. Input waveshape effect on delay for different transition time thresholds.

for the case when all of them switch together, are the two extreme cases of this family of curves. Consider the following scenario which illustrates the problem of threshold selection for multi-input gates. Suppose that the three inputs to the gate rise together with the same transition time. Based on our discussion for inverters, it is natural to measure delay using the $V_{i l}-V_{i h}$ thresholds obtained from the right most VTC (i.e. $a$, $b$, and $c$ tied together) in Figure 3-1(c). Now, consider a different situation; suppose inputs $b$ and $c$ switch together but input $a$ rises after $b$ and $c$ have finished their transitions. Since the last arriving input $a$ causes the output to change, we must measure delay using the $V_{i l}-V_{i h}$ thresholds obtained from the left most 


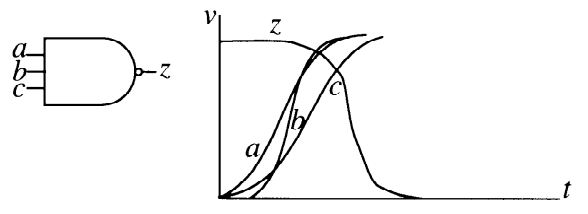

(a) Near-simultaneous switching in a multi-input gate

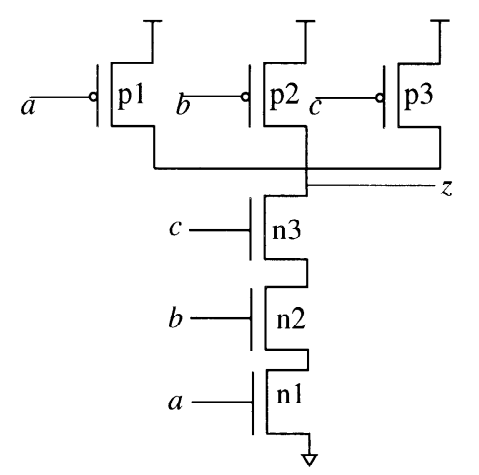

(b) Three-input NAND gate

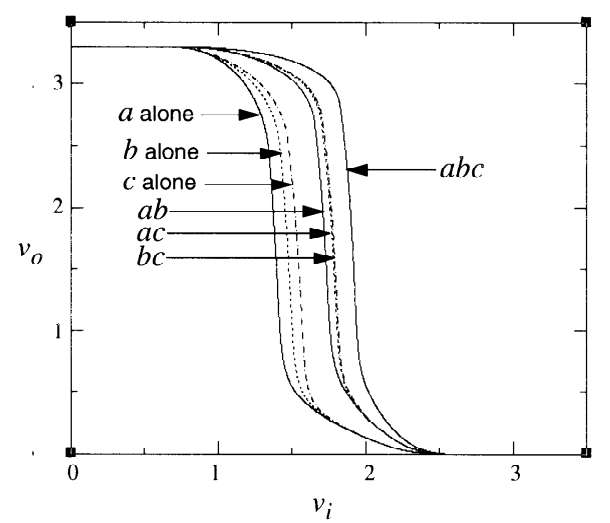

(c) VTCs of the NAND gate

\begin{tabular}{|c|c|c|c|}
\hline \hline input & $\begin{array}{c}V_{i l} \\
(\mathrm{~V})\end{array}$ & $\begin{array}{c}V_{m} \\
(\mathrm{~V})\end{array}$ & $\begin{array}{c}V_{i h} \\
(\mathrm{~V})\end{array}$ \\
\hline$a$ & $v_{1.065}$ & 1.401 & 1.585 \\
\hline$b$ & 1.161 & 1.481 & 1.665 \\
\hline$c$ & 1.239 & 1.545 & 1.718 \\
\hline$a, b$ & 1.482 & 1.723 & 2.078 \\
\hline$a, c$ & 1.542 & 1.772 & 2.085 \\
\hline$b, c$ & 1.555 & 1.78 & 2.093 \\
\hline$a, b, c$ & 1.723 & 1.9 & 2.246 \\
\hline \hline
\end{tabular}

(d) Various thresholds for the NAND gate

Fig. 3-1. Threshold selection for a 3-input NAND gate.

VTC (i.e. when $a$ switches alone). Note how we have to move from the thresholds of one VTC to the other VTC depending on the temporal separation of the inputs. It is not clear at what temporal separation of input $a$ from inputs $b$ and $c$ to make the transition from the right most VTC to the left most VTC in Figure 31(c). Instead, if we had continued to use the $V_{i l}-V_{i h}$ thresholds from the right-most VTC, then we can get negative delays when there is a large temporal separation between input $a$ and inputs $b$ and $c$ (and input $a$ is slow rising). This is so because $V_{i l}$ obtained from the VTC corresponding to the three inputs tied together, is greater than the $V_{m}$ obtained from the VTC corresponding to a switching alone, and as we saw in the 
inverter analysis (see (2.13)), this condition can yield negative delays.

To ensure that negative delays never arise and to avoid moving from one VTC to another depending on the separation of inputs, we base our delay measurement on the minimum $V_{i l}$ and the maximum $V_{i h}$ from all the VTCs. This will guarantee that $V_{i l}<V_{m}<V_{i h}$ for $V_{m}$ corresponding to any VTC and will therefore ensure positive delay, for any combination of input transition times and their temporal separations. In general, the lowest $V_{i l}$ would be from the VTC obtained by tying the inputs connected to the gates of the $n$-transistors closest to the ground rail. This is because the source of the transistors is at zero potential whereas the sources of other transistors higher in the series stack will have non-zero source voltage. Consequently a higher gate voltage $^{3}$ is required to turn on the transistors higher in the stack resulting in a higher value of $V_{i l}$. Similarly, the highest value of $V_{i h}$ would be from the VTC obtained by tying the inputs connected to the gates of the $p$-transistors closest to the power rail. Therefore, in case of a NAND gate, the $V_{i l}$ chosen would be from the input closest to the ground and $V_{i h}$ would be from the VTC corresponding to all inputs being tied together. In case of NOR gates, the situation is complementary, with $V_{i l}$ being chosen from the VTC corresponding to all inputs tied together and $V_{i h}$ being chosen from the input closest to the power rail. The $V_{i l}-V_{i h}$ thresholds used for delay and transition time measurement for the example NAND gate are shown shaded in Figure 3-1(d).

Complex Gates: Similar arguments hold in the case of complex gates (see Figure 3-2). While in case of simple gates it was easy to determine what combination of stable and switching inputs during the DC analysis would yield the minimum and maximum thresholds, in case of complex gates, this choice is topology dependent. The guiding principle is still the same: find the input combination that causes the output to fall at the earliest (for minimum $V_{i l}$ ) and the combination that causes the output to rise at the earliest (for maximum $\left.V_{i h}\right)$. For example, in the gate shown in Figure 3-2, the minimum $V_{i l}$ is determined by switching $x_{1}$ and $x_{2}$ together and setting $x_{3}$ and $x_{4}$ to 1 . Similarly, for the maximum $V_{i h}$ is determined by switching $x_{1}$ and $x_{4}$ together and setting $x_{3}$ and $x_{4}$ to 0 . This was further confirmed by a DC simulation of this gate.

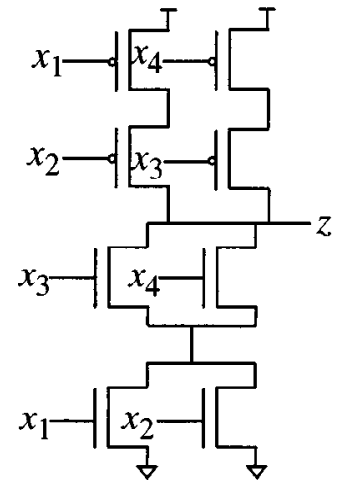

Fig. 3-2. Choosing thresholds for complex gates.

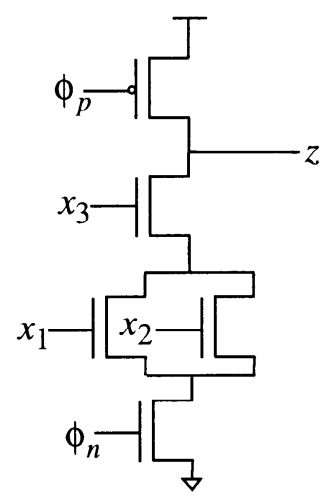

Fig. 3-3. Choosing the thresholds for a dynamic gate.

Dynamic Gates: In case of dynamic gates, there is just one pull-up transistor for the entire pulldown network (see Figure 3-3) and the inputs $\phi_{p}$ and $\phi_{n}$ are typically connected to the clock signal of the system. Therefore, we examine the topology of only the pulldown network to determine what inputs need to be switching and what inputs need to be stable high or low during the $\mathrm{DC}$ analysis to give the minimum $V_{i l}$ and the maximum $V_{i h}$. We then electrically connect $\phi_{p}$ to the input(s) in the pulldown network that are switching to get a valid VTC from which the thresholds can be found. For example, for the dynamic gate in Figure 3-3, we find through a DC simulation, that the minimum $V_{i l}$ is found from the VTC corresponding to inputs $x_{1}, x_{2}$ and $\phi_{p}$ switching together (with $x_{3}$ and $\phi_{n}$ stable high) and the maximum $V_{i h}$ is found from the VTC corresponding to $x_{1}, x_{2}, x_{3}, \phi_{n}$ and $\phi_{p}$ switching together.

This then completes our discussion of threshold selection for multi-input gates. To summarize, we find 
that unlike the inverter, we have not one but a family of VTCs for a multi-input gate. We therefore select the minimum and maximum of all possible $V_{i l}$ and $V_{i h}$ values respectively, as the thresholds for delay and transition time measurement. Based on the insight gained from the detailed study of inverters, we conclude that this choice always yields a positive value of delay for all input transition times and their temporal separations.

We note in passing that in a standard cell library based environment, there would be a wide variety of gates. For each gate in the library, we determine the $V_{i l}$ and $V_{i h}$ value using the ideas embodied in this paper. We then select the minimum of all the $V_{i l}$ values and the maximum of all the $V_{i h}$ values to be the delay and transition time thresholds for the entire system.

This concludes our analysis for logic gates. We next examine the problem of delay measurement for interconnect wires, since with the advent of deep submicron technologies, the temporal modeling of interconnect wires (or simply interconnects) is becoming a major concern.

\section{Threshold Selection for Interconnects}

In this paper, we shall confine the discussion to only point-to-point interconnect, such as shown in Figure 41(a). Unlike logic gates, interconnects are linear, passive (non-amplifying) circuits. Therefore, their VTC is a straight line with slope equal to 1 , as shown in Figure 4-1(b). ${ }^{4}$ For delay measurement, as in gates, once again we seek thresholds from the VTC to signify the beginning and end of events. However, we note that there are no clear-cut voltages which can demarcate events, since the interconnect VTC has a constant gain over the entire range of the input voltage (see Figure 4-1(c)). Moreover, referring to Figure 4-1(b), intuitively, it appears that the delay would approach zero as input transition time tends to infinity, for any choice of thresholds such that $V_{i t}=V_{o t}$, and that delay would never be negative (since interconnects are non-amplifying). However, since interconnects are not isolated circuits and are driven by buffer drivers having a well defined VTC, we use the unity differential gain thresholds (i.e. $V_{i l}-V_{i h}$ thresholds) of the driver for measuring delay and transition time thresholds. As shown in Figure 4-1(d), this leads to a consistent definition of delay for a combination of gate and interconnect.

In the following two subsections, we first investigate the behavior of delay with this choice of input thresholds for $R C$ interconnects and then examine the problem for $R L C$ interconnects.

\subsection{RC Interconnect}

For on chip wires, the inductive effects can be ignored and the interconnect can be treated as a distributed $R C$ network. In the following discussion, for simplicity, we consider only uniform distributed RC interconnect and approximate it by a lumped RC ladder circuit (see Figure 4-2(a)). In the figure, $r$ and $c$ are the per-unitlength resistance and capacitance, $l$ is the length of the interconnect and $n$ refers to the number of lumped segments. While $n$ should be sufficiently large for accuracy, for digital applications $n=5$ suffices in most cases [10].

We consider a saturated ramp input to the $n$-segment ladder network. It is well known that the poles of the transfer function of an $R C$-ladder are all distinct and lie on the negative real axis in the complex frequency domain [8]. Therefore, the impulse response of the ladder network in time domain can be written as follows:

$$
h(t)=\sum_{j=1}^{n} k_{j} e^{-\left|p_{j}\right|^{t}}
$$

where $p_{j}$ is the $j^{\text {th }}$ pole and $k_{j}$ is the corresponding residue. ${ }^{5}$ Since under DC conditions the output voltage equals the input voltage, we have the following relation between the poles and the residues:

$$
\sum_{j=1}^{n} \frac{k_{j}}{\left|p_{j}\right|}=1
$$

The output response of an $R C$ ladder is known to be monotonic [11] and is obtained by convolving the impulse response given by (4.1) with the ramp input. As shown in Figure 4-2(b), the output response has two parts and the crossover voltage is denoted by $V_{C}$. Since it was slow inputs that caused an anomalous behavior of delay in case of logic gates, we begin by examining the behavior of delay for a slow rising ramp input to an $R C$-ladder.

Slow inputs: We show that the delay approaches zero for any choice of threshold voltage such that $V_{i t}=V_{o t}$ as $\tau_{i} \rightarrow \infty$. It can be easily seen from the equation for $V_{C}$ in Figure 4-2(b) that the cross-over voltage $V_{C}$ is close to $V_{d d}$ in this case. Therefore we assume that the 


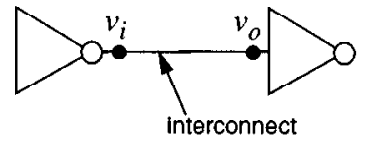

(a) Point-to-point interconnect

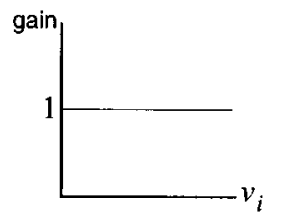

(c) Variation of gain

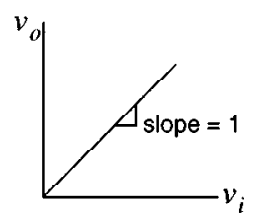

(b) VTC of interconnect

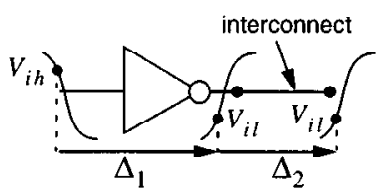

(d) Measuring delay for a combination of interconnect and gate

Fig. 4-1. Point-to-point interconnect and its VTC.

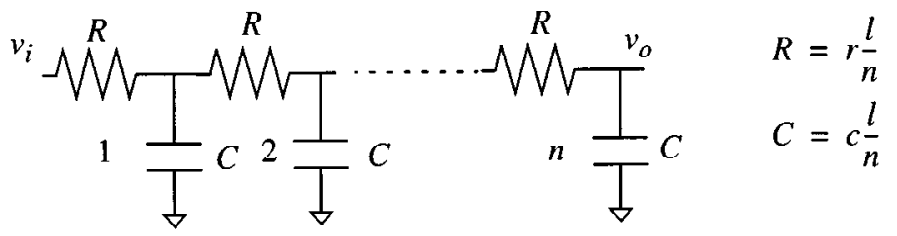

(a) Approximation of $R C$ interconnoct by $n$ lumped sections

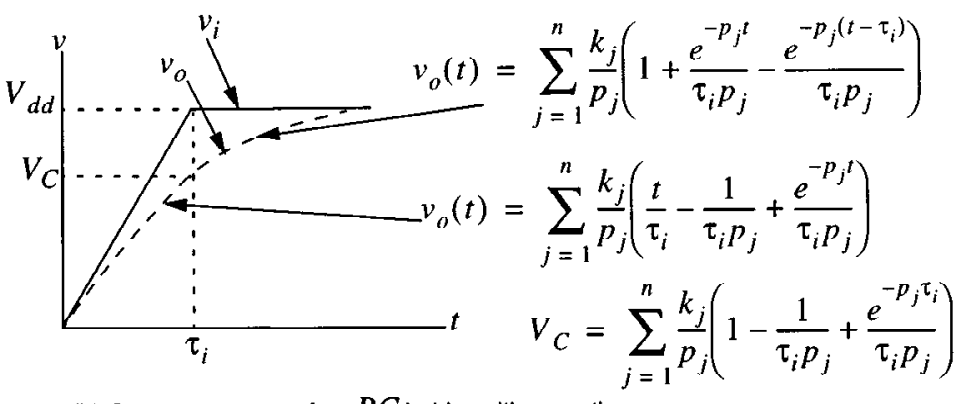

(b) Output response of an $R C$ ladiler wilh $n$ sections

Fig. 4-2. Lumped approximation of $R C$ interconnect.

output threshold is less than $V_{C}$ and the output voltage is then given by:

$$
v_{o}(t)=\sum_{j=1}^{n} \frac{k_{j}}{p_{j}}\left(\frac{t}{\tau_{i}}-\frac{1}{\tau_{i} p_{j}}+\frac{e^{-p_{j} t}}{\tau_{i} p_{j}}\right)
$$

Since this expression cannot be inverted for an explicit equation for delay, we resort to an indirect technique, by examining the output voltage at the instant the input crosses $V_{i t}$. The output voltage at this instant is given by:

$$
\begin{aligned}
v_{o}\left(\tau_{i} V_{i t}\right) & =\sum_{j=1}^{n} \frac{k_{j}}{p_{j}}\left(\frac{\tau_{i} V_{i t}}{\tau_{i}}-\frac{1}{\tau_{i} p_{j}}+\frac{1}{\tau_{i} p_{j} e^{p_{j} \tau_{i} V_{i t}}}\right) \\
& \cong V_{i t} \sum_{j=1}^{n} \frac{k_{j}}{p_{j}}=V_{i t}
\end{aligned}
$$

where use is made of the relation given by (4.2). Therefore, we conclude that delay approaches zero as the input gets slower. 
This is further confirmed experimentally using circuit simulation. Consider the circuit shown in Figure 43 (a) where a ramp voltage source (without loss of generality we use a ramp with $V_{l}=0 V$ and $V_{h}=1 V$ ) is driving an interconnect modeled by five lumped sections. The values for $R$ and $C$ are taken from [11]. In Figure 4-3(b) we plot $v_{o}$ at the instant $v_{i}$ crosses $V_{i t}$, as a function of the input transition time for three different threshold values (to mimic the different driver threshold values that could arise in practice). It is clear that the voltage at this instant approaches $V_{i t}$ as the input transition time is increased. Unlike gates, delay is never negative even for very slow inputs.

Fast inputs: In this case, $V_{C}$ is close to zero and the output voltage in the region of interest is given by:

$v_{o}(t)=\sum_{j=1}^{n} \frac{k_{j}}{p_{j}}\left(1+\frac{e^{-p_{j} t}}{\tau_{i} p_{j}}-\frac{e^{-p_{j}\left(t-\tau_{i}\right)}}{\tau_{i} p_{j}}\right)$

It is clear that the delay for a step input is positive since $v_{o}(0)$ is zero and it takes a finite amount of time for the output to reach the threshold. Unfortunately, it is not possible to infer the behavior (i.e. increasing or decreasing) of delay as a function of $\tau_{i}$ from (4.5). Instead, we experimentally determine the behavior of delay in Figure 4-3(c), where we plot delay versus the input transition time for relatively fast inputs. We find that delay increases with $\tau_{i}$ when $V_{i t}$ is less than or equal to the $50 \%$ threshold; however, it is interesting to note that it decreases when $V_{i t}$ is greater than this threshold. We note that, in general, $V_{\text {il (driver })}$ is less than the $50 \%$ threshold for most practical driver circuits and therefore the delay will increase with $\tau_{i}$ for fast inputs.

Thus, as suggested in the intuitive remarks in the beginning of Section 4, while delay never becomes negative, it does asymptote to zero as input transition time approaches infinity. In the next subsection we account for the inductive effects in the interconnect, since inductance can affect delay in board-level signal traces.

\subsection{RLC interconnect}

If the line resistance is small (which is usually the case for board level interconnect), we cannot approximate a uniform $R L C$ interconnect by a finite number of lumped elements for sufficient accuracy. Also, unlike $R C$ interconnect, the poles of the transfer function for an $R L C$ interconnect are not confined to the negative real axis and are complex. As a result the output could exhibit ringing as shown in Figure 4-4(a), which physically, is due to impedance mismatches in the interconnect circuit [2]. The logic stage connected to the output of the interconnect would then interpret the logic values as shown in Figure 4-4(b). While we define delay and signal transition time, as in the case of $R C$ interconnects, using the driver thresholds, we need an additional parameter, called the settling time (denoted by $\xi$ in the figure) to fully characterize the output from a temporal standpoint. From the figure we find that the output of the interconnect can be reliably latched only after $\Delta+\tau_{o}+\xi$. Using intuitive arguments based on the VTC and by an analysis similar to that of $R C$ interconnects, we can again show that $\Delta \rightarrow 0$ as $\tau_{i} \rightarrow \infty$ for any choice of thresholds such that $V_{i t}=V_{o t}$.

This completes our discussion of threshold selection for interconnects. In summary, we find that owing to the non-amplifying nature of interconnects, there is no particular voltage with which we can identify the occurrence of events. Hence, to consistently define delay for a combination of gates and interconnects, we use the $V_{i l}-V_{i h}$ thresholds from the VTC of the driver to measure the delay of the interconnect. We also showed that the delay asymptotes to zero as input transition time approaches infinity, for any choice of thresholds such that $V_{i t}=V_{o t}$. In case of $R L C$ interconnects, we needed an additional temporal parameter, called the settling time, to determine when the output became stable. In the following section, we conclude the paper by summarizing the important contributions of this research and placing them in the proper perspective.

\section{Conclusions}

In this paper, we have addressed the problem of threshold selection for measuring propagation delay and signal transition time in logic gates and interconnects. The central guiding theme in our study was that delay must be defined so that it reflects the cause and effect relationship between the input and output of a device. As we showed in the paper, non-conformity to this results in physically meaningless values of delay such as negative or zero delay.

We began our study by considering the threshold selection for single input gates. Through a logical, step-by-step development, beginning with a piecewiselinear input to an ideal inverter and concluding with circuit simulation using a realistic input to a physi- 
all resistances in ohms, capacitances in picofarads

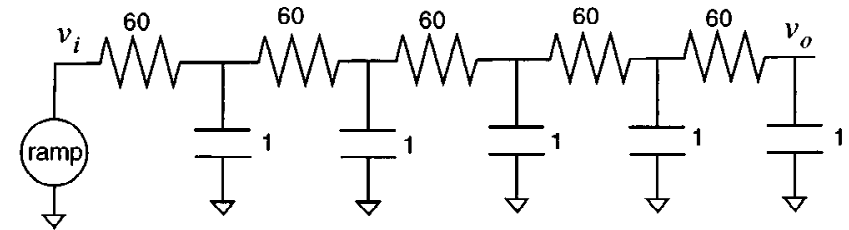

(a) An example $R C$ interconnect

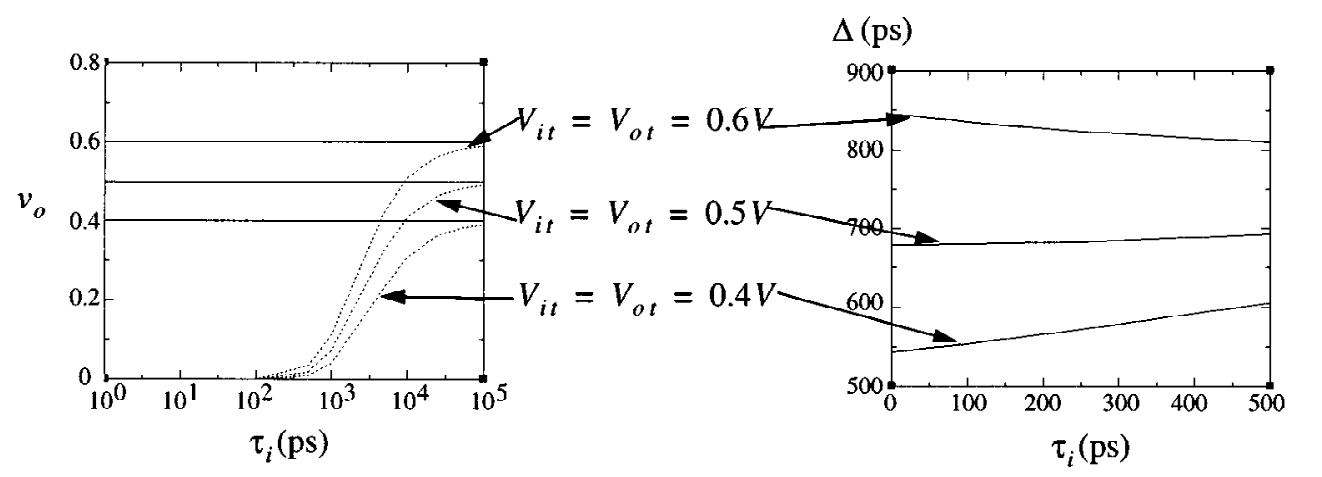

(b) Output voltage when input crosses threshold

(c) Behavior of delay for small transition times

Fig. 4-3. Circuit simulation of a lumped model of RC interconnect.

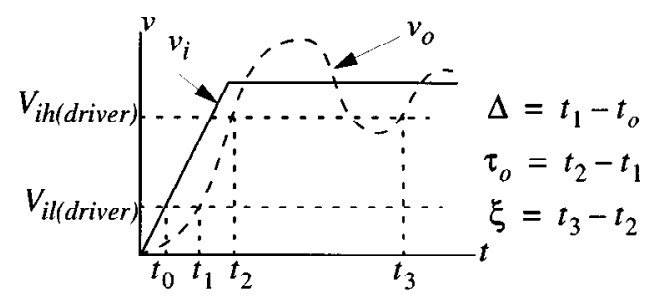

(a) Output response of an RLC interconnect

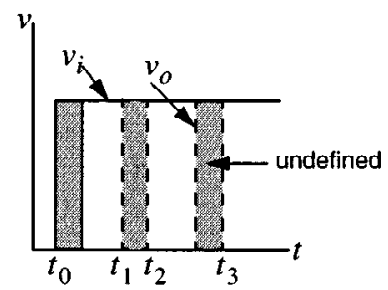

(b) Logic interpretation of output

Fig. 4-4. Output response of an $R L C$ interconnect.

cal inverter, we found that delay when measured using the unity differential gain thresholds, $V_{i l}$ and $V_{i h}$, from the inverter VTC, is always strictly positive. We also showed that when signal transition time is measured using these thresholds, the delay is relatively less sensitive to input waveshape effects, compared to the transition time measurement using the usual 10\%-90\% thresholds. Our analysis and subsequent experimental validation showed the inappropriateness of using the popular $50 \%$ threshold for delay measurement, since it can give rise to negative delays. We then examined the problem of threshold selection for multi-input gates, both static and dynamic. We found that in case of multi-input gates, we have not one but a family of dif- ferent VTCs corresponding to the various combination of switching and stable inputs. Based on the insight gained from the inverter study, we found that choosing the minimum $V_{i l}$ and the maximum $V_{i h}$ (from the family of VTCs) as the delay and transition time thresholds, ensured a strictly positive delay always. Finally, we examined the problem of threshold selection for simple, point-to-point interconnect structures. Owing to the passive nature of interconnects, we found that there was no clear-cut voltage from the interconnect VTC that would signify the start or end of input and output events. We also showed that while the delay does not become negative, it asymptotes to zero as input transition time approaches infinity for any choice 
of thresholds such that $V_{i t}=V_{o t}$. In order to define delay consistently for a combination of interconnects and gates, we found it necessary to use the $V_{i l}-V_{i h}$ thresholds of the gate driving the interconnect. In case of RLC interconnect, we discovered that the output response could be non-monotonic, and therefore we needed an additional parameter, the settling time, to fully characterize the output temporally.

We believe that this research is of fundamental importance to the accurate delay modeling of gates and interconnects. A careful definition of delay, such as outlined in this paper, paves a natural way for integrating the temporal and functional behavior of a logic gate. Such integrated models are necessary for an accurate and reliable timing analysis at the gate and system level. However, despite the theoretical and practical importance of correct delay measurement, we have not come across a comprehensive treatment of this problem in the literature. To the best of our knowledge, this is the first thorough study of the problem.

\section{Notes}

1. Note that in this case $V_{l}=0$ and $V_{h}=1$. However, any other choice of these thresholds would simply scale the transition time by a constant and would not affect the results of this and subsequent sections.

2. Curves similar to the transient VTC were shown in [13] and were called drive curves. However, they were derived using a model different from ours.

3. Another reason that necessitates a higher gate voltage is the body effect due to the non-zero source to bulk voltage.

4. Interconnects with shunt conductances would have a slope less than 1 ; however, in most practical interconnects, the shunt conductances are zero and hence we ignore shunt conductances in this paper.

5. These poles and residues can be found by moment matching methods such as AWE [19].

\section{References}

1. D. Aurvegne, N. Azemard, D. Deschacht, and M. Robert, "Input waveform slope effects in CMOS delays." IEEE Journal of Solid State Circuits 25(6), pp. 1588-1590, 1990.

2. H. B. Bakoglu, Circuits, interconnections, and packaging for VLSI. Addison-Wesley, 1990.

3. J. R. Burns, "Switching response of complementary-symmetry MOS transistor logic circuits." RCA Review 25(Dec), pp. 627661, 1964.

4. V. Chandramouli and K. A. Sakallah, "Modeling the effects of temporal proximity of input transitions on gate propagation delay and transition time." Proceedings 33rd IEEE/ACM Design Automation Conference pp. 617-622, 1996.
5. F. C. Chang, C. F. Chen, and P. Subramaniam, "An accurate and efficient gate level delay calculator for MOS circuits," in Proceedings 25th ACM/IEEE Design Automation Conference, pp. 282-287, 1988.

6. S. Dutta, S. S. M. Shetty, and S. L. Lusky, "A comprehensive delay model for CMOS inverters." IEEE Journal of Solid State Circuits 30(8), pp. 864-871, 1995.

7. W. C. Elmore, "The transient response of damped linear networks with particular regard to wide-band amplifiers." Journal of Applied Physics 19(1), pp. 55-63, 1948.

8. M. S. Ghaussi and J. J. Kelly, Introduction to Distributed Parameter Networks with Applications to Integrated Circuits. Hole, Rinehart, and Winston, Inc., 1968.

9. L. A. Glasser and D. W. Dobberpuhl, The Design and Analysis of VLSI Circuits. Addison-Wesley, 1985.

10. N. Gopal, D. P. Neikirk, and L. T. Pillage, "Evaluating $R C$ interconnect using moment-matching approximations," in $\mathrm{Di}$ gest of Technical Papers, ICCAD, pp. 74-777, 1991.

11. R. Gupta, B. Krauter, B. Tutuianu, J. Willis, and L. T. Pilegi, "The Elmore delay as a bound for RC trees with generalized input signals," in Proceedings 32nd IEEE/ACM Design Automation Conference, pp. 364-369, 1995.

12. Hewlett-Packard Document, CMOS 14tB Design Reference Manual, available through MOSIS.

13. M. A. Horowitz, "Timing models for MOS circuits," $\mathrm{PhD}$, Integrated Circuits Laboratory, Stanford University, 1984.

14. H. E. Kallman and R. E. Spencer, Proceedings of the IRE 33 , pp. 169-195, 1945.

15. A. I. Kayssi, K. A. Sakallah, and T. Mudge, "The impact of signal transition time on path delay computation." IEEE Transactions on Circuits and Systems-II: Analog and Digital Signal Processing 40(5), pp. 302-309, 1993.

16. E. Kreyszig, Advanced Engineering Mathematics. John Wiley and Sons, 1972.

17. Meta-Software, Hspice User's Manual. 1992.

18. H. N. Nham and A. K. Bose, "A multiple delay simulator for MOS LSI circuits." Proc. ACM/IEEE Design Automation Conference, pp. 610-617, 1980.

19. L. T. Pillage and R. A. Rohrer, "Asymptotic waveform evaluation for timing analaysis." IEEE Transactions on ComputerAided Design of Integrated Circuits and Systems 9(4), pp. 352366, 1990.

20. D. J. Pilling, P. F. Ordnung, and D. Heald, "Time delays in LSI circuits." Proc. IEEE Int'l Symposium on Circuit Theory, pp. 311-315, 1972.

21. T. Sakurai and A. R. Newton, "A simple MOSFET model for circuit analysis." IEEE Transactions on Electron Devices 38(4), pp. 887-894, 1991.

22. Wolfram Inc., Mathematica User's Manual, 2nd Edition.

V. Chandramouli (S'94) received the B.E. degree (with honors) in Computer Science and Technology 
from the University of Roorkee, Roorkee (India) in 1991 and the M.S. in Computer Science from the University of Utah in 1993. Since September, 1993, he has been a doctoral student in Computer Science and Engineering at the University of Michigan. His current research interests are in the area of circuit simulation, developing efficient timing and power models for gates and interconnects, timing analysis and the theory of music.

V. Chandramouli was the recipient of the University Merit Scholarship at Roorkee from 1988-1991 and is a student member of the IEEE.

Karem A. Sakallah (S'76-M'81-SM'92) received the B.E. degree (with distinction) in electrical engineering from the American University of Beirut, Beirut,
Lebanon, in 1975, and M.S.E.E. and Ph.D. degrees in electrical and computer engineering from Carnegie Mellon University, Pittsburgh, PA, in 1977 and 1981, respectively.

In 1981 he joined the Department of Electrical Engineering at CMU as a Visiting Assistant Professor. From 1982 to 1988 he was with the Semiconductor Engineering Computer-Aided Design Group at Digital Equipment Corporation in Hudson, Massachusetts, where he headed the Analysis and Simulation Advanced Development team. Since September 1988 he has been at the University of Michigan, Ann Arbor, MI, as Associate Processor of Electrical Engineering and Computer Science. From September 1994 to March 1995, he was on a six-month sabbatical leave at the Cadence Berkeley Laboratory in Berkeley, California. He is currently an Associate Editor for the IEEE Transactions on Computer-Aided Design of Integrated Circuits and Systems. His research interests are primarily in the area of computer-aided design, with particular emphasis on numerical analysis, multilevel simulation, timing verification and optimal clocking, modeling, knowledge abstraction, and design environments.

Karem Sakallah is a senior member of the IEEE and a member of the ACM and Sigma Xi. 